\title{
The potential impact of biomarker-guided triage decisions for patients with urinary tract infections
}

\author{
A. Litke $\cdot$ R. Bossart $\cdot$ K. Regez $\cdot$ U. Schild $\cdot$ \\ M. Guglielmetti $\cdot$ A. Conca $\cdot$ P. Schäfer · \\ B. Reutlinger $\cdot$ B. Mueller $\cdot$ W. C. Albrich
}

Received: 17 December 2012/ Accepted: 4 February 2013/Published online: 24 February 2013

(C) Springer-Verlag Berlin Heidelberg 2013

\begin{abstract}
Objectives Current guidelines provide limited evidence as to which patients with urinary tract infection (UTI) require hospitalisation. We evaluated the currently used triage routine and tested whether a set of criteria including biomarkers like proadrenomedullin (proADM) and urea have the potential to improve triage decisions.

Methods Consecutive adults with UTI presenting to our emergency department (ED) were recruited and followed for 30 days. We defined three virtual triage algorithms, which included either guideline-based clinical criteria, optimised admission proADM or urea levels in addition to a set of clinical criteria. We compared actual treatment sites and observed adverse events based on the physician judgment with the proportion of patients assigned to treatment sites according to the three virtual algorithms. Adverse outcome was defined as transfer to the intensive care unit (ICU), death, recurrence of UTI or rehospitalisation for any reason.

Results We recruited 127 patients (age $61.8 \pm 20.8$ years; $73.2 \%$ females) and analysed the data of 123 patients with a final diagnosis of UTI. Of these 123 patients, $27(22.0 \%)$ were treated as outpatients. Virtual triage based only on clinical signs would have treated only $22(17.9 \%)$ patients as outpatients, with higher proportions of outpatients equally in both biomarker groups $(29.3 \%$;
\end{abstract}

A. Litke $(\bowtie) \cdot$ B. Mueller $\cdot$ W. C. Albrich

Medical University Department of the University of Basel, Kantonsspital Aarau, Tellstrasse, 5001 Aarau, Switzerland

e-mail: alexander.litke@ksa.ch; alitke@gmx.ch

R. Bossart · K. Regez · U. Schild · M. Guglielmetti ·

A. Conca $\cdot$ P. Schäfer $\cdot$ B. Reutlinger

Department of Clinical Nursing Science, Kantonsspital Aarau,

Aarau, Switzerland $p=0.02$ ). There were no significant differences in adverse events between outpatients according to the clinical $(4.5 \%)$, proADM $(2.8 \%)$ or urea groups $(2.8 \%)$. The mean length of stay was 6.6 days, including 2.2 days after reaching medical stability.

Conclusions Adding biomarkers to clinical criteria has the potential to improve risk-based triage without impairing safety. Current rates of admission and length of stay could be shortened in patients with UTI.

Keywords Urinary tract infection · Triage $\cdot$ Biomarkers

\section{Introduction}

Urinary tract infection (UTI) is considered to be the most common bacterial infection in humans [1]. In adults aged 65 years or older, UTI is the second most common cause of infectious disease-related hospitalisations [2], with almost eight million annual consultations in emergency departments (EDs) in the USA alone [3]. The decision as to whether to admit a patient might be one of the most important clinical assessments made by physicians during the entire course of illness for patients with UTI. It has a direct influence on the intensity of laboratory testing, microbiological evaluation, antibiotic therapy and cost of treatment. Older patients who are hospitalised are more vulnerable to become impaired or lose their functional and self-care abilities, possibly leading to inpatient treatment or increased length of stay (LOS) [4]. Outpatient treatment, especially in elderly, fragile patients, is less expensive and carries a lower risk of hospital-acquired disability and nosocomial infections, including Clostridium difficileassociated diarrhoea [5]. Despite a low risk according to clinical severity scores, many patients with community- 
acquired infectious diseases are hospitalised for medical co-morbidities and psychosocial reasons [6, 7]. In a health system with a well-organised primary care system, patients with UTIs who were carefully selected for treatment at home according to clear guidelines did not have major complications [8].

The current guidelines for patients with UTI either lack specific criteria for the requirement of hospital admission and appropriateness of discharge or include fairly vague criteria and subjective parameters such as high fever, flank pain or severe malaise. These guidelines are based, rather, on expert opinion than strict medical evidence, with limited efficiency and safety profiles [9-15]. Most of these clinical criteria are fairly subjective and difficult to graduate or measure.

Biomarkers are objective, dynamic and easily measurable. Numerous studies have shown distinct evidence supporting the use of biomarkers to improve the diagnosis of bacterial infections and to guide antibiotic therapy [16-19]. Proadrenomedullin (proADM) was the most accurate biomarker for prognostic assessment [20, 21].

We recently reported improved performance of the combination of proADM with the prognostic CURB-65 score for predicting mortality and adverse outcomes in patients with lower respiratory tract infection (LRTI) compared to the CURB-65 score alone [22]. Biomarkerenhanced triage might have great potential to avoid notindicated hospital admissions and shorten the LOS [23].

Analogous studies do not yet exist for UTI, despite the apparent need for improved triage in daily clinical routine. Here, we extended our concept from LRTI to UTI and assessed the prognostic value of initial concentrations of proADM or urea in patients presenting to an ED with UTI. This was based on the hypothesis that the measurement of biomarkers provides additional prognostic information over clinical criteria alone regarding hospitalisation requirement. We also assessed whether the LOS for hospitalised patients with UTI could be shortened by applying objective stability criteria.

\section{Materials and methods}

Subjects and study design

This was a prospective observational quality control survey at the Kantonsspital Aarau, Switzerland, from January to September 2011. We consecutively enrolled all adults (age $\geq 18$ years) presenting to our ED with acute ( $<28$ days) symptoms typical for UTI. The inclusion criterion was an established diagnosis of UTI on admission. There were no exclusion criteria.
The triage, diagnostic and therapeutic judgments were taken by the treating physician without any influence by the study team, knowledge of virtual algorithms or of proADM levels. Patients were clinically observed from admission to discharge. Demographic, clinical and microbiological information was collected by the study team. Medical evaluation was performed daily in order to verify clinical stability. If a patient remained in hospital despite being medically stable, the physician in charge was asked to provide an overruling reason. Telephone interviews were performed with all patients 30 days after enrolment.

The local Institutional Review Board (Kantonale Ethikkommission Aargau) classified this study as an observational quality surveillance and waived the need for patient informed consent.

\section{Methods of proADM measurement}

ProADM was batch-measured in EDTA serum routinely collected on admission with a sandwich immunoassay (MR-ProADM, Thermo Fisher Scientific-BRAHMS AG, Hennigsdorf, Germany), with an analytical detection limit of $0.08 \mathrm{nmol} / \mathrm{l}$ and a functional assay sensitivity of $0.12 \mu \mathrm{g} / \mathrm{l}$ [24]. The results were not available at the time of hospitalisation and, thus, physicians and patients were blinded to these results.

\section{Definitions}

UTI was diagnosed in the presence of at least one clinical symptom of cystitis or pyelonephritis and at least one urinary criterion: $\geq 10^{5}$ colony-forming units $(\mathrm{CFU}) / \mathrm{ml}$ and $\leq 2$ different organisms in the culture (if not pretreated with antibiotics) [15], pyuria ( $>20$ leukocytes/ $\mu$ l) [25] or urine positive for leukocyte esterase and/or nitrites by dipstick [26]. Clinical symptoms of cystitis (lower UTI) comprised dysuria, frequency, urgency, suprapubic pain and/or hematuria [27]; signs and symptoms of pyelonephritis (upper UTI) comprised fever $\left(>38^{\circ} \mathrm{C}\right)$, chills, flank pain, costovertebral angle tenderness and nausea/vomiting, regardless of the presence of symptoms of cystitis [28, 29].

Uncomplicated UTI was restricted to female non-pregnant patients $<70$ years of age. Conversely, a complicated UTI was defined according to the literature $[10,12,14]$ as an infection associated with any condition or the presence of an underlying disease which increases susceptibility or reduces host response to infections: male sex, elderly age ( $>70$ years), hospital acquisition, pregnancy, indwelling urinary catheter, recent urinary tract intervention, functional or anatomical abnormality of the urinary tract, recent antimicrobial use, symptoms for $>7$ days at presentation, diabetes mellitus or immunosuppression. 
Using the above criteria, we distinguished four diagnoses: uncomplicated cystitis, complicated cystitis, uncomplicated pyelonephritis and complicated pyelonephritis. Patients were classified according to the final diagnosis and as 'other diagnosis' if the final diagnosis was different from UTI.

Based on a literature review, we chose the six most consistently mentioned hospitalisation criteria (Table 1). Medical stability was derived from the Infectious Diseases of American Society (IDSA) criteria for LRTI and adapted to UTI (Table 2). Patients were considered to be eligible for discharge from acute medical care if they were clinically stable for at least $24 \mathrm{~h}$ and did not require any further acute medical treatment.

Adverse outcomes included death, transfer to the intensive care unit (ICU), recurrence of UTI or rehospitalisation for any reason within 30 days of enrolment.

Independently of the actually observed treatment site allocation, every enrolled patient was retrospectively and virtually allocated to inpatient or outpatient treatment using three different triage algorithms (Fig. 1). The term 'virtual allocation' represents the treatment site (i.e. hospital admission or ambulant care) which should have been chosen according to the particular triage algorithm.

In the first virtual triage algorithm (guideline-concordant triage), all six admission criteria (Table 1) were used to assess the need for hospital admission. If any of these were present, inpatient treatment was recommended, otherwise outpatient treatment was following. This treatment site allocation was called 'clinical virtual triage' and all 'clinical virtual' inpatients and outpatients represented the 'clinical virtual group'.

While criteria 2-6 are objective, the first criterion in Table 1 (severe illness, high fever, costovertebral angle

Table 1 Criteria for admission

1. Severe illness, high fever, costovertebral angle tenderness, severely impaired health

2. Inability to take oral medications or fluids with dehydration

3. Questionable patient compliance

4. Pregnancy

5. Complications of pyelonephritis: papillary necrosis, intrarenal/ perirenal abscess, emphysematous pyelonephritis

6. Evidence of acute serious co-morbidity that necessitates hospitalisation

Table 2 Criteria for medical stability

1. Stable vital signs $\left(T \leq 37.8{ }^{\circ} \mathrm{C}\right.$, pulse $\leq 100 / \mathrm{min}$, respiratory

rate $\leq 24 / \mathrm{min}$, systolic blood pressure $\geq 90 \mathrm{mmHg}$ )

2. Feasibility of oral intake

3. Return to baseline mental status

4. No evidence of acute serious co-morbidity that necessitates hospitalisation tenderness, severely impaired health) appears subjective. To enhance the objectivity of the treatment site allocation, we replaced, in the second and third classifications, the first criterion with levels of the biomarkers proADM and urea, respectively. We determined the most suitable cut-off values using the best performance for both efficacy and safety.

In the second virtual triage algorithm ('proADM virtual triage'), proADM replaced the first hospitalisation criterion: if any of criteria 2-6 were present or proADM $\geq 1.5 \mathrm{nmol} / \mathrm{l}$, the patient was virtually allocated to inpatient care and otherwise to outpatient care. This classification was called 'proADM virtual triage' and the patients represented the 'virtual proADM group'.

In the third virtual triage algorithm ('urea virtual triage'), urea was used instead of the first admission criterion: if any of criteria $2-6$ were present or urea $\geq 14 \mathrm{mmol} / \mathrm{l}$, the patient was virtually allocated to inpatient care and otherwise to outpatient care. This classification was called 'urea virtual triage' and the patients represented the 'urea virtual group'.

The selection of treatment sites made in real life by the physician in charge was called 'actual triage' and the patients represented the 'actual treatment group'. This real group and the three virtual ones contain the same number of patients (equal to the number of enrolled patients), but the distribution of inpatient and outpatient treatment between the groups vary.

\section{Endpoints}

Our primary endpoint was to compare the percentages of patients eligible for outpatient treatment between the three virtual triage algorithms. Secondary endpoints were the determination of hospitalisation length before and after reaching medical stability and prediction of adverse events by the different triage algorithms.

Statistical analysis

Discrete variables were expressed as counts (percentage) and continuous variables as medians or means and standard deviations or interquartile range, as appropriate. The comparison between qualitative variables was performed by Fisher's exact test or the Mantel-Haenszel $\chi^{2}$ test, as appropriate, and for quantitative normally distributed data by Student's $t$-test. For data not normally distributed, but with a similar shape, the Mann-Whitney $U$-test was used. Statistical analyses were performed using SAS $\left(\mathrm{SAS}^{\circledR}\right.$ Institute Inc., Cary, NC, USA) and Epi Info (version 3.5.1, CDC, Atlanta, GA, USA). All testing was two-tailed and $p$-values $<0.05$ were considered to indicate statistical significance. 
Fig. 1 Algorithm for virtual triage decisions on admission

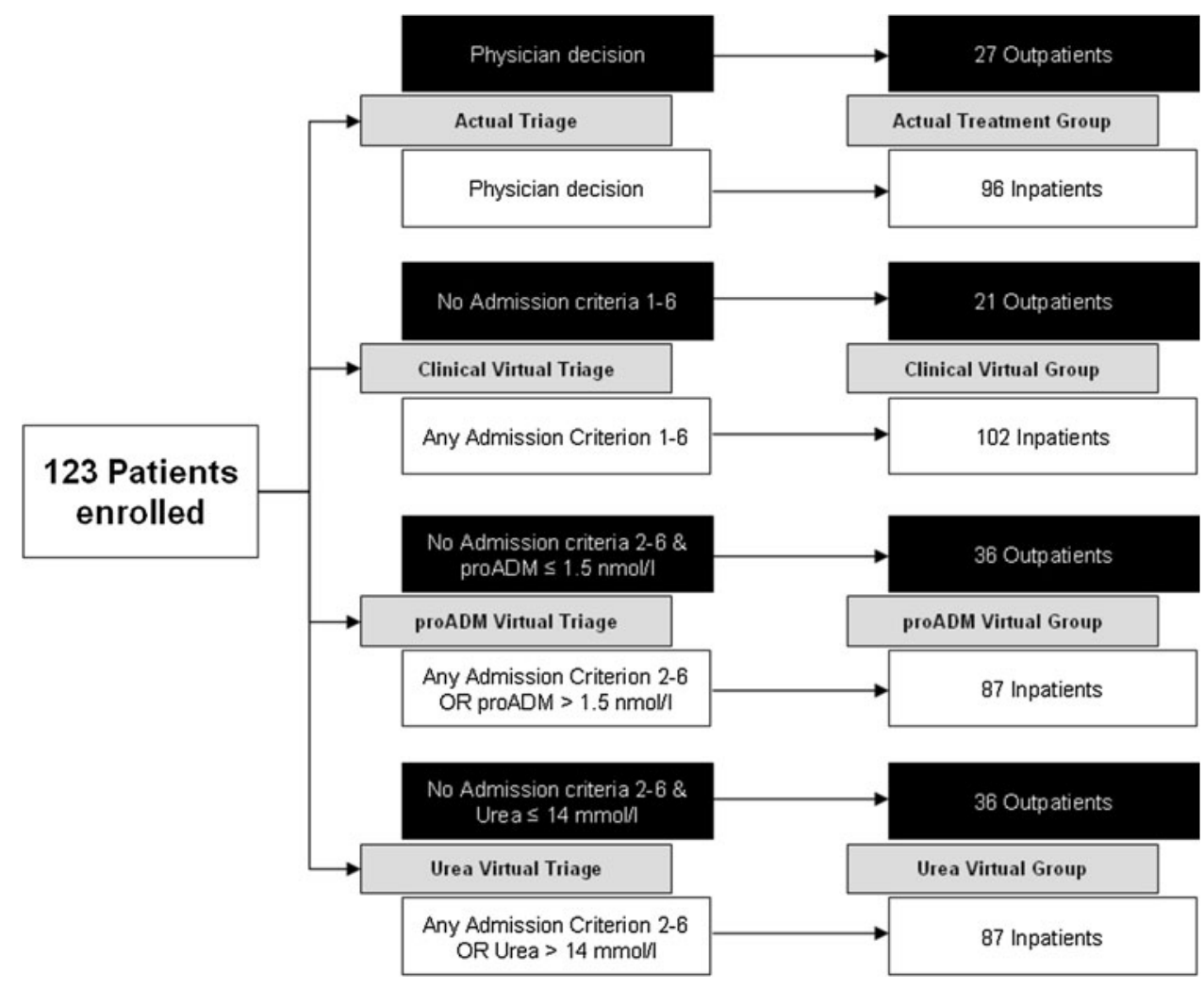

\section{Results}

Baseline characteristics

A total of 127 patients (mean age $61.8 \pm 20.8$ years; $73.2 \%$ females) were enrolled in our study. Their baseline characteristics are shown in Table 3. The 123 patients with a final diagnosis of UTI, comprising nine with uncomplicated and 32 with complicated cystitis, 20 with uncomplicated and 62 with complicated pyelonephritis, were further analysed. Twenty-seven patients $(22 \%)$ were treated as outpatients and $96(78 \%)$ were hospitalised. The distribution of hospitalisation criteria is shown in Table 4 . The serum levels of biomarkers on admission were significantly higher in inpatients than in outpatients (median proADM 1.25 vs. $0.53 \mathrm{nmol} / 1, p<0.001$, median urea 6.4 vs. $4.5 \mathrm{mmol} / \mathrm{l}, p<0.01$ ) (Fig. 2).

\section{Allocation of the virtual treatment site}

According to only clinical criteria ('clinical virtual group'), 21 of 123 patients $(17.1 \%)$ would qualify for outpatient treatment and $102(82.9 \%)$ should be admitted to the hospital. Applying the 'proADM virtual triage' or 'urea virtual triage', $36(29.3 \%)$ patients would not require hospitalisation and $87(70.7 \%)$ patients should be hospitalised (Fig. 3). As shown in Table 5, 10 of 36 (27.8\%) patients who would be eligible for outpatient treatment according biomarker-enhanced triage were admitted to the hospital in real life.

Comparing the virtual algorithms, the 'proADM virtual triage' and 'urea virtual triage' would have allowed significantly more outpatient treatments than would have occurred using 'clinical virtual triage' ( $p=0.02$ for both, Fig. 3).

The difference in the proportion of outpatients between 'actual triage' and 'proADM virtual triage' with respect to 'urea virtual triage' was not statistically significant $(p=0.19$, Fig. 3).

\section{Microbiological data and antibiotic use}

The results of urine cultures were available for 120 patients (98\%). The most common pathogen was Escherichia coli (58\%), followed by Klebsiella species (5\%). Mixed flora was found in $6 \%$ of cultures and, in $19 \%$ of all cases, there was no significant bacteriuria; $74 \%$ of the latter were pre-treated with antibiotics. The spectra of pathogens for inpatients and outpatients were similar (Table 6).

Blood cultures on admission were performed for 82 patients $(67 \%)$. In the case of admission, $77 \%$ of patients underwent blood culture sampling with $15 \%$ having bacteraemia. Blood cultures were performed in $30 \%$ of outpatients, with only one positive result (4\%).

All patients were treated with an antibiotic. Fluoroquinolones were used for 19 patients $(15.7 \%)$, penicillins 


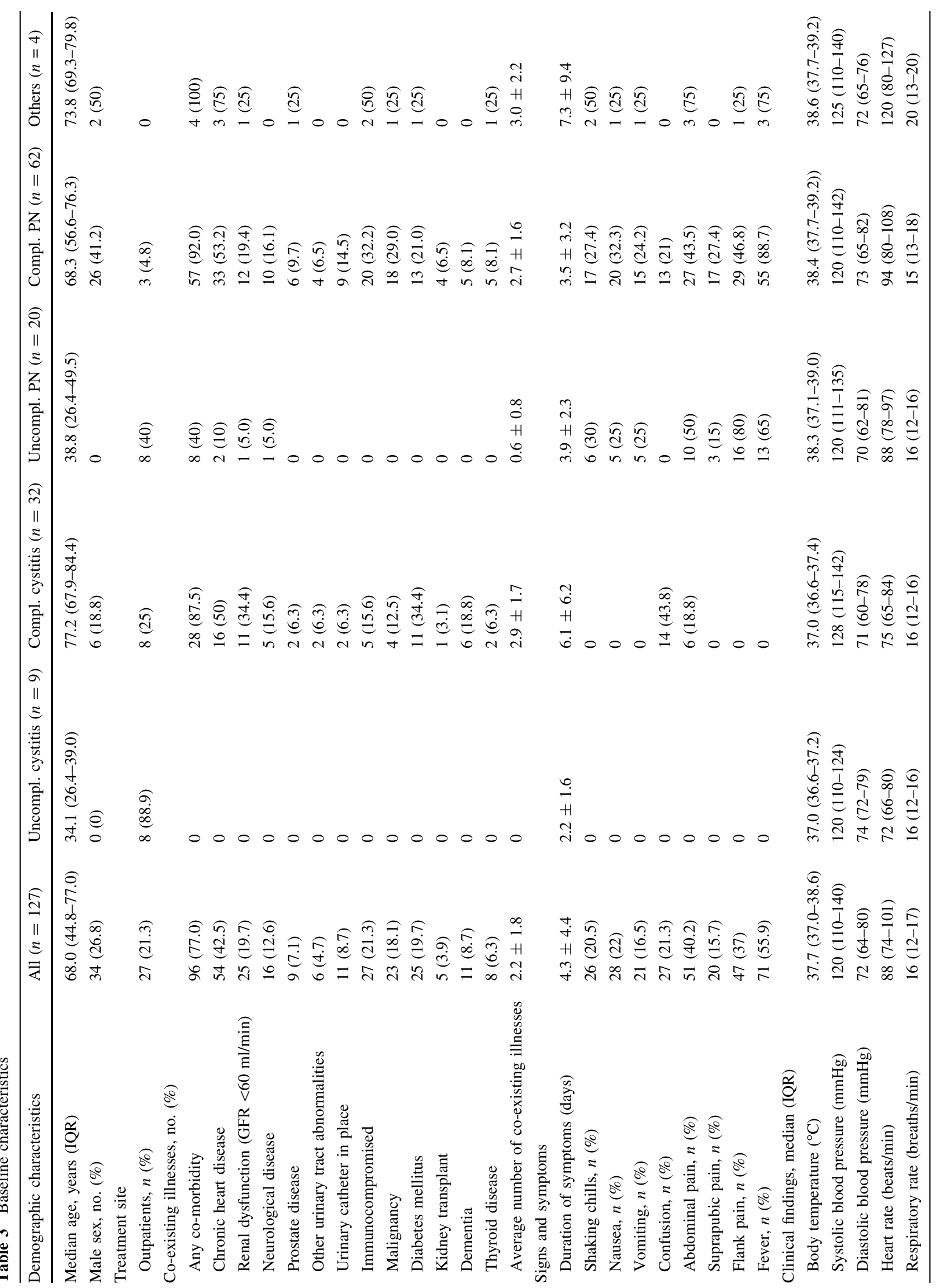




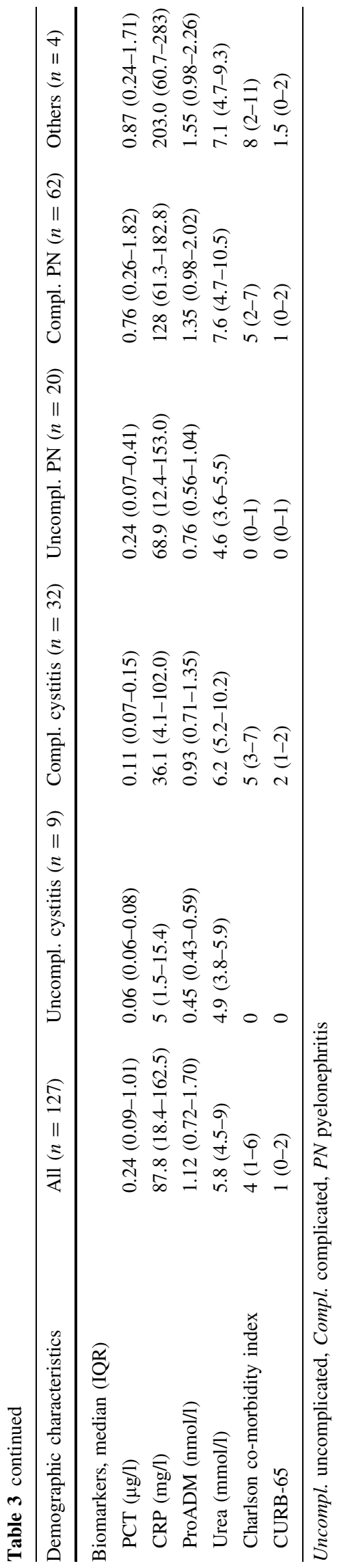

for 19 patients $(15.7 \%)$, cephalosporins for 60 patients $(48.8 \%)$ and trimethoprim-sulphamethoxazole for 25 patients $(20.3 \%)$. Forty-four patients $(35.8 \%)$ were initially treated orally and the other $79(64.2 \%)$ received at least one antibiotic dose intravenously. Looking at inpatients and outpatients separately, significantly $(p<0.001)$ more inpatients were treated intravenously compared to outpatients: 75 of 96 (78.1\%) versus 4 of 27 (14.8\%).

All four outpatients who received intravenous antibiotics and eight of the inpatients were given a single shot of ceftriaxone, followed by other antibiotics orally.

Length of stay and time to medical stability

Patients who were admitted to the hospital stayed for, on average, 6.6 days in the hospital. The mean time to medical stability was 4.4 days; thus, patients remained hospitalised for 2.2 days after reaching medical stability. All patients treated as outpatients in real life left the emergency department medically stable.

\section{Adverse events}

Complications were present in 25 (20.3\%) of 123 patients; the breakdown according to the different virtual triages is shown in Table 7. Four patients (3.3\%), all of them treated as inpatients, died. All of them would have been triaged to inpatient care according to all three virtual triage algorithms.

There were significantly $(p=0.02)$ fewer adverse events encountered in patients treated at home $(3.7 \%)$ than in hospitalised patients $(25 \%)$. There were no statistically significant differences in the adverse events between outpatients according to the clinical $(4.8 \%)$, proADM $(2.8 \%)$ or urea groups $(2.8 \%)$. We found no statistically significant differences for adverse events between real life and virtual triages with biomarkers. Using the virtual algorithms with proADM or urea, the difference in the number of adverse events between outpatients and inpatients was statistically significant $(p=0.002)$, but this was not the case in the clinical virtual group $(p=0.08)$.

The admission levels of proADM were significantly higher in patients with an adverse event within 30 days than those without (median 1.67 vs. $1.00 \mathrm{nmol} / 1, p=$ $0.003)$, whereas this difference was not significant for urea (median 6.4 vs. $5.6 \mathrm{mmol} / \mathrm{l}, p=0.267$ ) (Fig. 4).

\section{Discussion}

Our primary endpoint was to investigate the potential impact of using biomarkers for the decision regarding hospital admission in patients with UTI. Herein, we present four key findings. First, both proADM and urea levels were 
Table 4 Distribution of criteria for hospitalisation

\begin{tabular}{|c|c|c|c|c|c|}
\hline Hospitalisation criteria & $\begin{array}{l}\text { All } \\
(n=123)\end{array}$ & $\begin{array}{l}\text { Uncompl. } \\
\text { Cyst. }(n=9)\end{array}$ & $\begin{array}{l}\text { Compl. Cyst. } \\
(n=32)\end{array}$ & $\begin{array}{l}\text { Uncompl. PN } \\
(n=20)\end{array}$ & $\begin{array}{l}\text { Compl. PN } \\
(n=62)\end{array}$ \\
\hline $\begin{array}{l}\text { 1. Severe illness, high fever, costovertebral angle tenderness, } \\
\text { severely impaired health }\end{array}$ & 83 & 0 & 10 & 16 & 57 \\
\hline 2. Inability to take p.o. medications or fluids with dehydration & 80 & 1 & 17 & 11 & 51 \\
\hline 3. Questionable patient compliance & 4 & 0 & 1 & 1 & 2 \\
\hline 4. Pregnancy & 0 & 0 & 0 & 0 & 0 \\
\hline $\begin{array}{l}\text { 5. Complications of pyelonephritis: papillary necrosis, intrarenal/ } \\
\text { perirenal abscess, emphysematous pyelonephritis }\end{array}$ & 0 & 0 & 0 & 0 & 0 \\
\hline $\begin{array}{l}\text { 6. Evidence of acute serious co-morbidity that necessitates } \\
\text { hospitalisation }\end{array}$ & 32 & 0 & 16 & 2 & 14 \\
\hline
\end{tabular}

Uncompl. uncomplicated, Compl. complicated, Cyst. cystitis, $P N$ pyelonephritis

Fig. 2 Distribution of biomarkers on admission for inpatients and outpatients. The lower and upper limits of the boxes indicate the 1 st and $3 \mathrm{rd}$ quartiles, respectively; the horizontal line in the box represents the median value
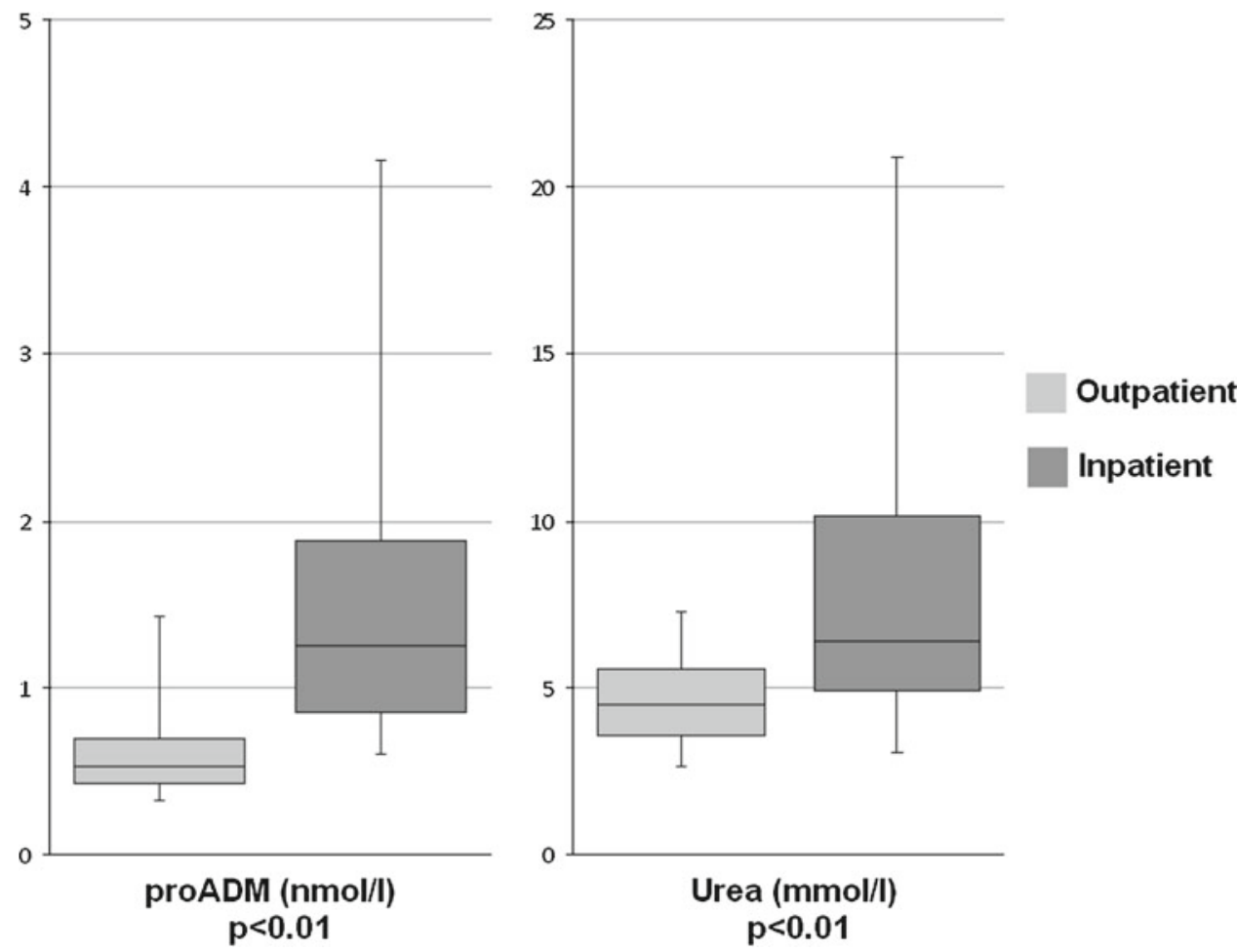

higher for inpatients than outpatients. Second, only proADM levels were higher in those patients with adverse events compared to those without. Third, as compared to current guidelines, using either proADM or urea instead of rather subjective hospitalisation criteria as part of a clinical algorithm would have allowed a higher proportion of patients to be safely treated as outpatients. Finally, we demonstrate that patients with UTI are hospitalised for more than 2 days after they have reached medical stability.

The combination of proADM or urea with clinical criteria increases the proportion of outpatients compared to current triage practice, and even more so if the current guidelines had been strictly adhered to. This result was achieved without an increase of adverse events. Another $12 \%$ of patients could be treated at home using biomarkerenhanced triage.

The hospitalisation rate of $78 \%$ among our patients was higher than that reported by Claessens et al. [30] and in the study of Elkharrat et al. [31], where only 26 and $13 \%$ of patients, respectively, were admitted. The main reason for this difference was likely the fact that our cohort was markedly older and sicker. In the two previous studies, the mean age for outpatients was 33 or 34.1 years and for inpatients, it was 46 or 55.7 years, respectively. Our outpatients had a median age of 41 years and our inpatients had a median age of 72 years, respectively, and 33 and 


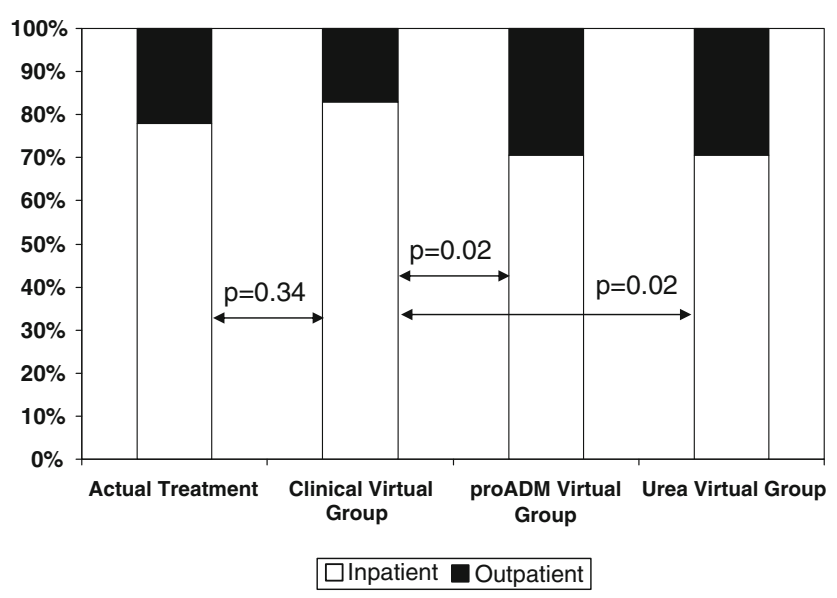

Fig. 3 Site of care by actual triage and the virtual triage algorithms

Table 5 Distribution of actual and suggested virtual treatment sites

\begin{tabular}{lll}
\hline & Inpatient & Outpatient \\
\hline Clinical virtual group & & \\
Suggested virtual treatment site: no. (\%) & $102(82.9)$ & $21(17.1)$ \\
Actual treatment site: inpatient/outpatient & $94 / 8$ & $2 / 19$ \\
proADM virtual group & & \\
Suggested virtual treatment site: no. (\%) & $87(70.7)$ & $36(29.3)$ \\
Actual treatment site: inpatient/outpatient & $86 / 1$ & $10 / 26$ \\
Urea virtual group & & \\
Suggested virtual treatment site: no. (\%) & $87(70.7)$ & $36(29.3)$ \\
Actual treatment site: inpatient/outpatient & $86 / 1$ & $10 / 26$ \\
\hline & &
\end{tabular}

$88 \%$ had at least one co-morbidity, respectively, which was more than in the other studies. This picture corresponds to the situation in the EDs of well-developed countries where patients are becoming older and have more impaired health [32, 33]. Another reason for the high hospitalisation rate might have been the preselection made by family physicians, who decided whether to refer the patient to an ED or not. In Switzerland, many patients consult the family physician first; thus, most patients who can be treated in the ambulant setting never present to the ED.

In a comparable study of van Nieuwkoop et al. [8] (similar population with a median age 64 years, $61 \%$ with co-morbidities), $86 \%$ of patients presenting to the ED were hospitalised using triage which was based only on clinical criteria. Using our clinical virtual algorithm, the proportion of inpatients would be comparable at $83 \%$, while more patients would have been treated outside the hospital using biomarker-enhanced triage in our study.

We found only a non-significant trend for less inpatient assignment with the biomarker virtual triage (71\%) compared to our current actual triage $(78 \%)$. This finding could be based on the fact that our physicians in charge had already participated in a similar trial for optimised triage in LRTI [23] and, thus, were sensitized for increasing the outpatient ratio. Furthermore, our hospital performs regularly at or beyond maximal bed capacity, leading to frequent bed shortages and more restrictive hospitalisations than recommended by the guidelines.

The 30-day mortality rate in our study is similar to recently reported rates $[8,34]$. The overall mortality was $3 \%$ : all outpatients stayed alive, whereas $4 \%$ of inpatients died. We found no differences for adverse events between real life and virtual triages with biomarkers. In the ProHOSP study [35], the fear of adverse outcome outside of hospital was one of the most important reasons for hospital admission for LRTI. None of the patients who would have been assigned ambulatory care according to our proposed

Table 6 Urine culture findings

\begin{tabular}{|c|c|c|c|c|c|c|c|}
\hline $\begin{array}{l}\text { Uropathogen: } \\
\text { no. }(\%)\end{array}$ & $\begin{array}{l}\text { All patients } \\
(n=123)(\%)\end{array}$ & $\begin{array}{l}\text { Inpatients } \\
(n=96)(\%)\end{array}$ & $\begin{array}{l}\text { Outpatients } \\
(n=27)(\%)\end{array}$ & $\begin{array}{l}\text { Uncompl. Cyst. } \\
(n=9)(\%)\end{array}$ & $\begin{array}{l}\text { Compl. Cyst. } \\
(n=32)(\%)\end{array}$ & $\begin{array}{l}\text { Uncompl. PN } \\
(n=20)(\%)\end{array}$ & $\begin{array}{l}\text { Compl. PN } \\
(n=62)(\%)\end{array}$ \\
\hline E. coli & $71(58)$ & $56(58)$ & $15(56)$ & $4(44)$ & $17(53)$ & $16(80)$ & $34(55)$ \\
\hline Klebsiella spp. & $6(5)$ & $6(6)$ & 0 & 0 & 0 & 0 & $6(10)$ \\
\hline $\begin{array}{l}\text { Enterococcus } \\
\text { faecalis }\end{array}$ & $3(2)$ & $2(2)$ & $1(4)$ & 0 & $1(3)$ & 0 & $2(3)$ \\
\hline $\begin{array}{c}\text { Pseudomonas } \\
\text { aeruginosa }\end{array}$ & $2(2)$ & $2(2)$ & 0 & 0 & 0 & 0 & $2(3)$ \\
\hline $\begin{array}{l}\text { Citrobacter } \\
\text { spp. }\end{array}$ & $2(2)$ & $1(1)$ & $1(4)$ & 0 & $1(3)$ & 0 & $1(2)$ \\
\hline $\begin{array}{l}\text { Streptococcus } \\
\text { spp. }\end{array}$ & $2(2)$ & $2(2)$ & 0 & 0 & $2(6)$ & 0 & 0 \\
\hline Other & $4(3)$ & $3(3)$ & $1(4)$ & $1(11)$ & 0 & 0 & $3(5)$ \\
\hline Mixed flora & $7(6)$ & $6(6)$ & $1(4)$ & $1(11)$ & $3(9)$ & 0 & $3(5)$ \\
\hline No growth & 23 (19) & 18 (19) & $5(19)$ & $1(11)$ & $8(25)$ & $3(15)$ & $11(18)$ \\
\hline
\end{tabular}

Uncompl. uncomplicated, Compl. complicated, Cyst. cystitis, $P N$ pyelonephritis 
Table 7 Adverse events stratified for the different virtual triage algorithms

\begin{tabular}{|c|c|c|c|c|c|c|c|c|c|}
\hline & \multicolumn{2}{|c|}{ Actual treatment group } & \multicolumn{2}{|c|}{ Clinical virtual group } & \multicolumn{2}{|c|}{ ProADM virtual group } & \multicolumn{2}{|c|}{ Urea virtual group } & \multirow{2}{*}{$\begin{array}{l}\text { Overall } \\
(n=123)\end{array}$} \\
\hline & $\begin{array}{l}\text { Outpatient } \\
(n=27)\end{array}$ & $\begin{array}{l}\text { Inpatient } \\
(n=96)\end{array}$ & $\begin{array}{l}\text { Outpatient } \\
(n=21)\end{array}$ & $\begin{array}{l}\text { Inpatient } \\
(n=102)\end{array}$ & $\begin{array}{l}\text { Outpatient } \\
(n=36)\end{array}$ & $\begin{array}{l}\text { Inpatient } \\
(n=87)\end{array}$ & $\begin{array}{l}\text { Outpatient } \\
(n=36)\end{array}$ & $\begin{array}{l}\text { Inpatient } \\
(n=87)\end{array}$ & \\
\hline Death: no. (\%) & 0 & $4(4.2)$ & 0 & $4(3.9)$ & 0 & $4(4.6)$ & 0 & $4(4.6)$ & $4(3.3)$ \\
\hline $\begin{array}{l}\text { ICU admission: } \\
\text { no. }(\%)\end{array}$ & 0 & $4(4.2)$ & 0 & $4(3.9)$ & 0 & $4(4.6)$ & 0 & $4(4.6)$ & $4(3.3)$ \\
\hline Relapse: no. (\%) & $1(3.7)$ & $12(12.5)$ & $1(4.8)$ & $12(11.8)$ & $1(2.8)$ & $12(13.8)$ & $1(2.8)$ & $12(13.8)$ & $13(10.6)$ \\
\hline $\begin{array}{l}\text { Rehospitalisation: } \\
\text { no. }(\%)\end{array}$ & 0 & $10(10.4)$ & 0 & $10(9.8)$ & 0 & $10(11.5)$ & 0 & $10(11.5)$ & $10(8.1)$ \\
\hline Related to UTI & 0 & $4(4.2)$ & 0 & $4(3.9)$ & 0 & $4(4.6)$ & 0 & $4(4.6)$ & $4(3.3)$ \\
\hline Unrelated to UTI & 0 & $6(6.3)$ & 0 & $6(5.9)$ & 0 & $6(6.9)$ & 0 & $6(6.9)$ & $6(4.9)$ \\
\hline Any: no. (\%) & $\begin{array}{l}1(3.7) \\
p=0.02\end{array}$ & $24(25)$ & $\begin{array}{l}1(4.8) \\
p=0.08\end{array}$ & $24(23.5)$ & $\begin{array}{l}1(2.8) \\
p=0.002\end{array}$ & $24(27.6)$ & $\begin{array}{l}1(2.8) \\
p=0.002\end{array}$ & $24(27.6)$ & $25(20.3)$ \\
\hline
\end{tabular}

Fig. 4 Distribution of biomarkers on admission for patients with and without adverse events. The lower and upper limits of the boxes indicate the 1st and 3rd quartiles, respectively; the horizontal line in the box represents the median value
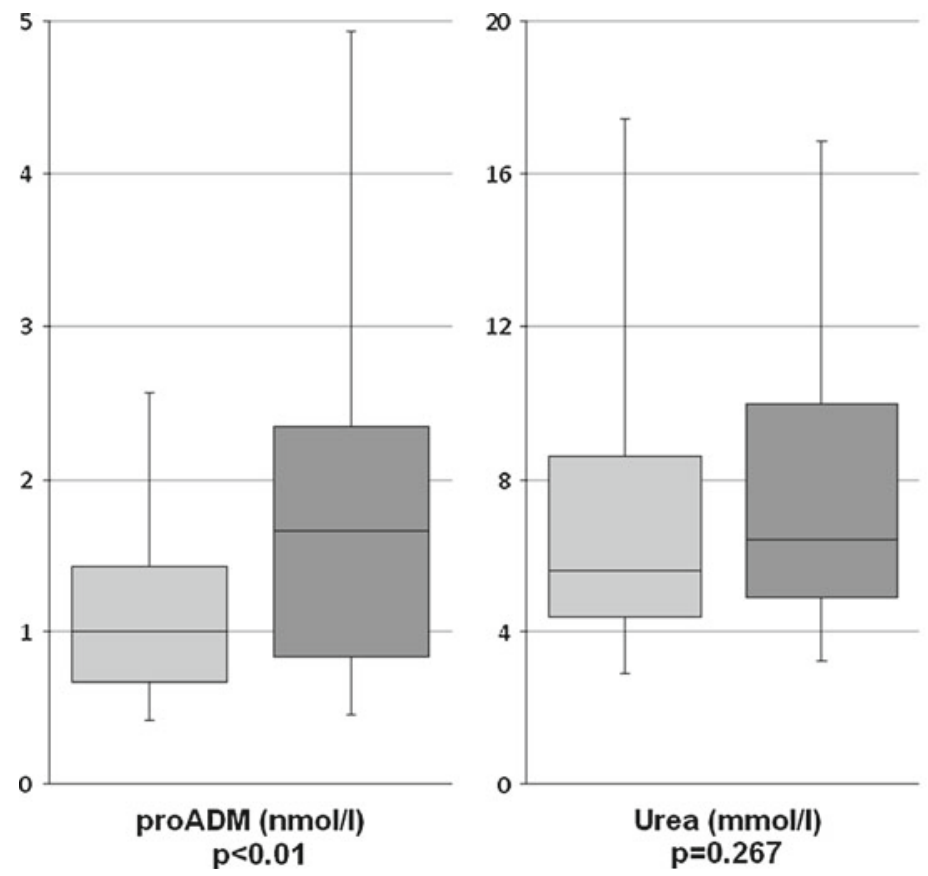

No adverse events

Adverse events biomarker-enhanced triage experienced death, ICU admission or rehospitalisation, and only 1 (2.8\%) of the proposed outpatients had a relapse. While it might be argued that these adverse events might have been prevented by the actual inpatient care, the observation of higher proADM levels in those patients with adverse outcomes supports the prognostic utility of proADM. Despite formally failing to achieve significant differences between patients with and those without adverse events for urea levels, a urea-enhanced algorithm would have triaged patients identically to a proADM-based triage. As urea is more affected by renal function, proADM might be more useful in patients with chronic renal insufficiency. The adverse events rate between inpatients and outpatients in the clinical virtual group was similar, suggesting that the triage based only on clinical criteria might be inferior to biomarkers in distinguishing between high-risk and lowrisk patients. Biomarkers alone also do not reliably help physicians in their decision-making process of the requirement for admission, even though neither urea nor proADM were evaluated in that study by Claessens et al. [30]. Conversely, adding biomarkers to clinical criteria, i.e. combining the two powerful physicians' instruments, could improve the prognostic assessment.

We also demonstrated that the LOS for hospitalised patients could be substantially shortened. The mean length 
of hospitalisation for our population was 6.6 days. In the recent work published by Elkharrat et al. [31], the mean LOS was 19 days. We observed that patients stayed 2.2 days longer after being medically stable. Thus, the stability criteria should be regularly assessed throughout the hospitalisation (e.g. daily) in order to facilitate a timely discharge and avoidance of unnecessarily longer hospitalisation. Prolonged hospitalisations have been associated with increased risk of nosocomial infections, falls, medication side effects and worsening or new onset of frailty [4].

There are limitations of this observational survey. We collected data from only one hospital and, so, the external validity of the results may be limited. We computed a composite of adverse events as an endpoint and chose the composite to create clinically meaningful outcome in light of sample size considerations. The triages being compared were virtual. This study design gave us the opportunity to evaluate the different triages using a significantly smaller sample size. The outcome comparison is hypothetical as well. As the classes and the route of application of antibiotics differ significantly between inpatients and outpatients, on first sight, it appears difficult to draw conclusions on the virtual outcome. Thirty-six patients would be eligible for outpatient treatment according to the virtual triage with biomarkers. Twenty-six of them were outpatients in real life and ten were admitted to the hospital. Four of the former and two of the latter $(p=0.74)$ received a single shot of ceftriaxone in the ED and, thereafter, the regimen was switched to oral antibiotics. Such a course of therapy was shown to be safe in different studies [9, 10, 31]. Since the remaining majority of 22 outpatients and eight inpatients were treated orally from the beginning and only a single dose of antibiotic given intravenously, the patients in these two subgroups were comparable in terms of antibiotic therapy. Importantly, this strategy is highly possible for outpatient care, as indicated in our study. Therefore, the route of antibiotic application itself would not have made outpatient therapy impossible, which would confirm our conclusion that outpatient treatment was, indeed, underutilised. Despite the absence of a significant difference in antibiotic use, there are still discrepancies (e.g. observation, nursing care, nutrition, risk of infection etc.) in terms of the general management between the 'real' hospital and 'virtual' home.

The knowledge from this survey provides the basis for an ongoing interventional clinical trial with serially measured proADM, where the different triage pathways are tested in real life. We did not separate patients according to different entities of UTI but used the same triage algorithm for all of them. Of note, more patients with pyelonephritis (87\%) than cystitis $(61 \%)$ were admitted.

One of the strengths of our study is the innovative idea of biomarker-enhanced triage for UTI. We have shown that biomarkers can be used not only for the diagnosis-making process, but also could help to decide whether the patient should be admitted or not. We included all consecutive patients without exclusion criteria in order to make our results applicable to an unrestricted patient population. We were also able to follow-up all patients for 30 days.

In conclusion, there is potential for biomarker-enhanced triage to avoid not-indicated hospital admissions without impairing safety. The addition of biomarkers to clinical criteria might increase confidence in objective triage decisions compared to clinical criteria alone.

As urea is more affected by renal function, proADM might be more useful in patients with chronic renal insufficiency. The LOS might be shortened if clinical stability criteria are assessed daily.

Acknowledgments We are grateful to all the physicians, nurses, social workers and patients who participated in this study. Especially, we thank Petra Tobias and the staff of the emergency room, Susanne Schirlo and the staff of the medical clinic, Renate Hunziker, Martha Kaeslin and the central laboratory of the Kantonsspital Aarau for their very helpful assistance and technical support.

Conflict of interest Werner Albrich and Beat Mueller received support from BRAHMS Thermo Fisher and from bioMérieux to attend meetings and fulfilled speaking engagements and served as consultants for BRAHMS Thermo Fisher. Beat Müller received research support from BRAHMS Thermo Fisher. All other authors report no conflict of interest.

\section{References}

1. Nicolle LE. Epidemiology of urinary tract infections. Infect Med. 2001;18:153-62.

2. Curns AT, Holman RC, Sejvar JJ, Owings MF, Schonberger LB. Infectious disease hospitalizations among older adults in the United States from 1990 through 2002. Arch Intern Med. 2005;165:2514-20.

3. Blondeau JM. Current issues in the management of urinary tract infections: extended-release ciprofloxacin as a novel treatment option. Drugs. 2004;64:611-28.

4. Covinsky KE, Pierluissi E, Johnston CB. Hospitalization-associated disability: "She was probably able to ambulate, but I'm not sure". JAMA. 2011;306:1782-93.

5. Chalmers JD, Al-Khairalla M, Short PM, Fardon TC, Winter JH. Proposed changes to management of lower respiratory tract infections in response to the Clostridium difficile epidemic. J Antimicrob Chemother. 2010;65:608-18.

6. Aliyu ZY, Aliyu MH, McCormick K. Determinants for hospitalization in "low-risk" community acquired pneumonia. BMC Infect Dis. 2003;3:11.

7. Labarere J, Stone RA, Scott Obrosky D, et al. Factors associated with the hospitalization of low-risk patients with communityacquired pneumonia in a cluster-randomized trial. J Gen Intern Med. 2006;21:745-52.

8. van Nieuwkoop C, van't Wout JW, Spelt IC, et al. Prospective cohort study of acute pyelonephritis in adults: safety of triage towards home based oral antimicrobial treatment. J Infect. 2010;60:114-21. 
9. Warren JW, Abrutyn E, Hebel JR, Johnson JR, Schaeffer AJ, Stamm WE. Guidelines for antimicrobial treatment of uncomplicated acute bacterial cystitis and acute pyelonephritis in women. Infectious Diseases Society of America (IDSA). Clin Infect Dis. 1999;29:745-58.

10. Bass PF 3rd, Jarvis JA, Mitchell CK. Urinary tract infections. Prim Care. 2003;30:41-61, v-vi.

11. Hooton TM. The current management strategies for communityacquired urinary tract infection. Infect Dis Clin North Am. 2003; 17:303-32.

12. Ramakrishnan K, Scheid DC. Diagnosis and management of acute pyelonephritis in adults. Am Fam Physician. 2005;71:933-42.

13. Kim K, Lee CC, Rhee JE, et al. The effects of an institutional care map on the admission rates and medical costs in women with acute pyelonephritis. Acad Emerg Med. 2008;15:319-23.

14. Hooton TM. Clinical manifestations; diagnosis; and treatment of acute pyelonephritis. In: Calderwood SB, Baron EL, editors. UpToDate. Retrieved from http://www.uptodate.com/home/ index.html.

15. Grabe M, Bjerklund-Johansen TE, Botto H, Çek M, Naber KG, Tenke P, Wagenlehner F. Guidelines on urological infections. European Association of Urology (EAU) guidelines. Edition presented at the 25th Anniversary EAU Congress, Barcelona, Spain, April 2010.

16. Nanda N, Juthani-Mehta M. Novel biomarkers for the diagnosis of urinary tract infection - a systematic review. Biomark Insights. 2009;4:111-21.

17. Schuetz P, Albrich W, Mueller B. Procalcitonin for diagnosis of infection and guide to antibiotic decisions: past, present and future. BMC Med. 2011;9:107.

18. Chalupa $\mathrm{P}$, Beran $\mathrm{O}$, Herwald $\mathrm{H}$, Kaspř́íková N, Holub M. Evaluation of potential biomarkers for the discrimination of bacterial and viral infections. Infection. 2011;39:411-7.

19. Lipinska-Gediga M, Mierzchala M, Durek G. Pro-atrial natriuretic peptide (pro-ANP) level in patients with severe sepsis and septic shock: prognostic and diagnostic significance. Infection. 2012;40:303-9.

20. Christ-Crain M, Morgenthaler NG, Struck J, Harbarth S, Bergmann A, Müller B. Mid-regional pro-adrenomedullin as a prognostic marker in sepsis: an observational study. Crit Care. 2005;9:R816-24.

21. Schuetz P, Wolbers M, Christ-Crain M, et al. Prohormones for prediction of adverse medical outcome in community-acquired pneumonia and lower respiratory tract infections. Crit Care. 2010;14:R106.

22. Albrich WC, Dusemund F, Rüegger K, et al. Enhancement of CURB65 score with proadrenomedullin (CURB65-A) for outcome prediction in lower respiratory tract infections: derivation of a clinical algorithm. BMC Infect Dis. 2011;11:112.
23. Albrich WC, Rüegger K, Dusemund F, et al. Optimised patient transfer using an innovative multidisciplinary assessment in Kanton Aargau (OPTIMA I): an observational survey in lower respiratory tract infections. Swiss Med Wkly. 2011;141:w13237.

24. Morgenthaler NG, Struck J, Alonso C, Bergmann A. Measurement of midregional proadrenomedullin in plasma with an immunoluminometric assay. Clin Chem. 2005;51:1823-9.

25. Regeniter A, Haenni V, Risch L, et al. Urine analysis performed by flow cytometry: reference range determination and comparison to morphological findings, dipstick chemistry and bacterial culture results-a multicenter study. Clin Nephrol. 2001;55: 384-92.

26. Kouri T, Fogazzi G, Gant V, Hallander H, Hofmann W, Guder WG. European urinalysis guidelines. Scand J Clin Lab Invest Suppl. 2000;60:1-96.

27. Bent S, Nallamothu BK, Simel DL, Fihn SD, Saint S. Does this woman have an acute uncomplicated urinary tract infection? JAMA. 2002;287:2701-10.

28. Fairley KF, Carson NE, Gutch RC, et al. Site of infection in acute urinary-tract infection in general practice. Lancet. 1971;2:615-8.

29. Scholes D, Hooton TM, Roberts PL, Gupta K, Stapleton AE, Stamm WE. Risk factors associated with acute pyelonephritis in healthy women. Ann Intern Med. 2005;142:20-7.

30. Claessens YE, Schmidt J, Batard E, et al. Can C-reactive protein, procalcitonin and mid-regional pro-atrial natriuretic peptide measurements guide choice of in-patient or out-patient care in acute pyelonephritis? Biomarkers In Sepsis (BIS) multicentre study. Clin Microbiol Infect. 2010;16:753-60. doi:10.1111/j. 1469-0691.2009.02955.x.

31. Elkharrat D, Chastang C, Boudiaf M, Le Corre A, Raskine L, Caulin C. Relevance in the emergency department of a decisional algorithm for outpatient care of women with acute pyelonephritis. Eur J Emerg Med. 1999;6:15-20.

32. Roberts DC, McKay MP, Shaffer A. Increasing rates of emergency department visits for elderly patients in the United States, 1993 to 2003. Ann Emerg Med. 2008;51:769-74.

33. Businger AP, Kaderli R, Burghardt LR, Osinga R, Liesch M, Furrer M. Demographic changes and their implications in a nonacademic emergency department in Switzerland: an 11-year trend analysis (2000-2010) of 104,510 patients. ISRN Emerg Med. 2012:Article ID 865861;2012. doi:10.5402/2012/865861.

34. Foxman B, Klemstine KL, Brown PD. Acute pyelonephritis in US hospitals in 1997: hospitalization and in-hospital mortality. Ann Epidemiol. 2003;13:144-50.

35. Baehni C, Meier S, Spreiter P, et al. Which patients with lower respiratory tract infections need inpatient treatment? Perceptions of physicians, nurses, patients and relatives. BMC Pulm Med. 2010;10:12. 